\title{
Tissue-restricted genome editing in vivo specified by microRNA-repressible anti-CRISPR proteins
}

\author{
JOOYOUNG LEE, ${ }^{1}$ HAIWEI MOU, ${ }^{1,4}$ RAED IBRAHEIM, ${ }^{1}$ SHUN-QING LIANG, ${ }^{1}$ PENGPENG LIU, ${ }^{2}$ WEN XUE, ${ }^{1,3}$ \\ and ERIK J. SONTHEIMER ${ }^{1,3}$ \\ ${ }^{1}$ RNA Therapeutics Institute, University of Massachusetts Medical School, Worcester, Massachusetts 01605, USA \\ ${ }^{2}$ Program in Molecular, Cell and Cancer Biology, University of Massachusetts Medical School, Worcester, Massachusetts 01605, USA \\ ${ }^{3}$ Program in Molecular Medicine, University of Massachusetts Medical School, Worcester, Massachusetts 01605, USA
}

\begin{abstract}
CRISPR-Cas systems are bacterial adaptive immune pathways that have revolutionized biotechnology and biomedical applications. Despite the potential for human therapeutic development, there are many hurdles that must be overcome before its use in clinical settings. Some clinical safety concerns arise from editing activity in unintended cell types or tissues upon in vivo delivery (e.g., by adeno-associated virus (AAV) vectors). Although tissue-specific promoters and serotypes with tissue tropisms can be used, suitably compact promoters are not always available for desired cell types, and AAV tissue tropism specificities are not absolute. To reinforce tissue-specific editing, we exploited anti-CRISPR proteins (Acrs) that have evolved as natural countermeasures against CRISPR immunity. To inhibit Cas9 in all ancillary tissues without compromising editing in the target tissue, we established a flexible platform in which an Acr transgene is repressed by endogenous, tissue-specific microRNAs (miRNAs). We demonstrate that miRNAs regulate the expression of an Acr transgene bearing miRNA-binding sites in its $3^{\prime}$-UTR and control subsequent genome editing outcomes in a cell-type specific manner. We also show that the strategy is applicable to multiple Cas9 orthologs and their respective anti-CRISPRs. Furthermore, we validate this approach in vivo by demonstrating that AAV9 delivery of Nme2Cas9, along with an AcrlIC $3_{\mathrm{Nme}}$ construct that is targeted for repression by liver-specific miR-122, allows editing in the liver while repressing editing in an unintended tissue (heart muscle) in adult mice. This strategy provides safeguards against off-tissue genome editing by confining Cas9 activity to selected cell types.
\end{abstract}

Keywords: Cas9; anti-CRISPR; microRNA; tissue-specific editing; AAV

\section{INTRODUCTION}

Clustered, regularly interspaced, short, palindromic repeats (CRISPR) and CRISPR-associated (cas) genes comprise prokaryotic adaptive immune defense systems that are organized into two major classes and multiple types and subtypes (e.g. II-A, -B, and -C) (Makarova et al. 2018). Cas9s are monomeric effector proteins in Type II systems that can target nearly any DNA sequence when guided by a CRISPR RNA (crRNA) base paired with a trans-activating RNA (tracrRNA), or as a fused form of both RNAs known as single guide RNA (sgRNA) (Garneau et al. 2010; Deltcheva et al. 2011; Jinek et al. 2012). The robustness and ease of Cas9 programmability

\footnotetext{
${ }^{4}$ Present address: Cold Spring Harbor Laboratory, Cold Spring Harbor, NY 11724, USA

Corresponding authors: wen.xue@umassmed.edu, erik. sontheimer@umassmed.edu

Article is online at http://www.rnajournal.org/cgi/doi/10.1261/rna. 071704.119.
}

have greatly facilitated its rapid adoption in genome editing and modulation (Komor et al. 2017). As medical, agricultural, and environmental technologies advance, safety concerns must be considered and addressed, especially with potential human therapeutics. In vivo therapeutics will often require not only precise editing at the intended genomic site but also in the intended tissue, given the possible risks of unwanted double-strand break (DSB) induction. For example, Cas9-induced DSBs can elicit translocations that can be associated with heritable disorders or various kinds of cancer, or large deletions and other rearrangements (Maddalo et al. 2014; Jiang et al. 2016; Kosicki et al. 2018). Moreover, some delivery modalities such as viral vectors are likely to affect many

(C) 2019 Lee et al. This article is distributed exclusively by the RNA Society for the first 12 months after the full-issue publication date (see http://rnajournal.cshlp.org/site/misc/terms.xhtml). After 12 months, it is available under a Creative Commons License (Attribution-NonCommercial 4.0 International), as described at http:// creativecommons.org/licenses/by-nc/4.0/. 
cell types and tissues beyond the intended therapeutic target (Hinderer et al. 2018). Adeno-associated virus (AAV) is currently the most widely used transgene delivery vector for therapeutic applications in preclinical and clinical settings. Different AAV serotypes have some tissue tropism, however, they can still infect broad ranges of tissues in vivo (Gao et al. 2004). Although tissuespecific promoters can be used to drive transgene expression in particular cell types (Walther and Stein 1996), some target tissues lack promoters that are sufficiently active, specific, or small for AAV deployment. These limitations necessitate the development of new regulatory strategies to enforce tissue specificity for in vivo applications.

Although several means of regulating genome editing activities have been reported, a prominent recent advance has resulted from the discovery of anti-CRISPR (Acr) proteins (Bondy-Denomy et al. 2013). Acrs are small proteins encoded by bacteriophages and other mobile genetic elements that have evolved as natural countermeasures against CRISPR-Cas immunity. Type II Acrs targeting Cas9 orthologs (Pawluk et al. 2016; Hynes et al. 2017, 2018; Rauch et al. 2017; Uribe et al. 2019), as well as the recently discovered Type V Acrs targeting Cas12a (Marino et al. 2018; Watters et al. 2018), are of particular interest because they can potentially provide temporal, spatial, or conditional control over established genome editing systems. Applications of Acrs have been demonstrated in bacteria (Rauch et al. 2017; Marshall et al. 2018), in yeasts to inhibit gene drives (Basgall et al. 2018), and in mammalian cells to modulate genome editing, dCas9based imaging, epigenetic modification, and genetic circuits (Pawluk et al. 2016; Rauch et al. 2017; Shin et al. 2017; Bubeck et al. 2018; Liu et al. 2018; Nakamura et al. 2019).

To improve current technologies that regulate the tissue specificity of editing, we have developed an Acr-based approach to inhibit Cas9 in all ancillary tissues while allowing editing in the target tissue. To spatially regulate $\mathrm{Acr}$ expression, we exploited endogenous tissue-specific microRNAs (miRNAs) to repress Acr expression in the target tissue. MiRNAs are a class of small regulatory RNAs whose mechanisms of messenger RNA (mRNA) regulation are extensively studied (Jonas and Izaurralde 2015). These RNAs load into an Argonaute protein (e.g., Ago2) to form RNA-induced silencing complexes (RISCs) that recognize complementary sequences present in mRNA targets, leading to translational repression and mRNA destabilization (Bartel 2018). In mammalian cells, Ago2-loaded miRNAs can subject extensively or perfectly complementary mRNA targets to endonucleolytic cleavage, enabling strong down-regulation. Since miRNA response elements (MREs) are very small ( $22 \mathrm{nt}$ or less), this regulatory modality places a minimal burden on AAV vector capacity, which is limited to $\sim 4.8 \mathrm{~kb}$. Moreover, large numbers of mammalian cell and tissue types express specific combinations of tissue-restricted miRNAs (Lagos-Quintana et al. 2002).

Here we establish a flexible platform in which an $\mathrm{Acr}$ transgene is repressed by endogenous, tissue-specific miRNAs to control Acr expression spatially. We demonstrate that miRNAs can regulate the expression of an $\mathrm{Acr}$ transgene bearing miRNA-binding sites in its $3^{\prime}$ untranslated region (UTR) and control subsequent genome editing outcomes in a cell-type specific manner. We also show that the strategy is applicable to multiple Cas9 orthologs and their respective Acrs, including the widely used Streptococcus pyogenes Cas9 (SpyCas9) (Cho et al. 2013; Cong et al. 2013; Hwang et al. 2013; Jinek et al. 2013; Mali et al. 2013) as well as the more readily AAVdeliverable Cas9 orthologs from Neisseria meningitidis (Nme1Cas9 and Nme2Cas9) (Edraki et al. 2018; Ibraheim et al. 2018). Furthermore, we have expressed antiCRISPR proteins in mice to achieve efficient inhibition of Cas9-mediated genome editing in vivo without detectable toxicity. Most importantly, we show that codelivery of Nme2Cas9, guide RNA, and miR-122-repressible AcrllC3 $3_{\text {Nme }}$ transgenes by AAV9 vectors in adult mice allow editing in the liver (the only tissue where miR-122 is expressed) but suppress editing in an ancillary tissue (heart). This strategy establishes the in vivo efficacy of Acrs in mammals and provides the basis for restriction of undesired off-tissue editing by confining Cas9 activity to selected cell types.

\section{RESULTS}

\section{AAV delivery of Nme1Cas9 and sgRNA results in editing in various tissues}

Previously, our group has used all-in-one AAV8 to deliver a human-codon-optimized Nme1Cas9 for genome editing in vivo (Ibraheim et al. 2018). Nme1Cas9 is smaller and less prone to off-target editing than the widely used SpyCas9 (Amrani et al. 2018). Upon delivery of allin-one AAV8 vectors expressing Nme1Cas9 driven by a ubiquitous U1a promoter and sgRNA via tail vein injection, we observed high editing efficiency in liver tissues collected $50 \mathrm{~d}$ post-injection (Ibraheim et al. 2018). To gauge editing efficiencies in nontarget tissues of these same mice (previously unpublished data), samples from cardiac and skeletal muscle (gastrocnemius) as well as kidney and brain were collected and analyzed in the current study (Supplemental Fig. S1). Although off-tissue editing efficiencies were lower than those observed in the liver $(57.8 \pm 8.5 \%)$, appreciable indel frequencies were observed in different organs, especially in the heart $(22.5 \pm 5.2 \%)$ (Supplemental Fig. S1). This is consistent with previous reports that AAV8 effectively transduces mouse hepatocytes but also infects skeletal and cardiac 
muscles (Nakai et al. 2005) as well as brain at high doses (Zincarelli et al. 2008). These observations, along with the known multitissue tropisms of other AAV serotypes (Zincarelli et al. 2008), underscore the need for systems that confine Cas9 activity to selected tissues.

\section{A strategy for microRNA- regulated anti-CRISPR proteins}

Endogenous miRNA-mediated posttranscriptional gene silencing has proven to be an effective and tissuespecific approach to regulate transgene expression upon AAV delivery in vivo (Xie et al. 2011). Delivery of Cas9 and sgRNA via AAV has the potential to induce editing in multiple transduced tissues (e.g., heart, skeletal muscle, and elsewhere); however, codelivery of the miRNA-repressible Acr will inhibit editing in such nontarget tissues due to the latter's lack of particular tissue-specific miRNAs (and therefore their inability to silence the expression of the Acr inhibitor). In the case of the liver-specific miRNA miR-122, in the target tissue, the Acr gene with miR-122 MREs will be repressed, enabling Cas9-mediated editing (Fig. 1A). In contrast, off-tissue editing (e.g., in cardiac and skeletal muscle, Supplemental Fig. S1) will be inhibited by the Acr, since those extrahepatic tissues lack miR-122 and therefore fail to silence Acr expression. To validate this concept, we chose two well-established Cas9Acr combinations: $\mathrm{Acrll}_{\mathrm{C}} 3_{\mathrm{Nme}}$ and Nme1Cas9 or Nme2Cas9 (Type II-C; Pawluk et al. 2016; Edraki et al. 2018) as well as AcrllA $4_{\text {Lmo }}$ and SpyCas9 (Type II-A; Rauch et al. 2017). Nme2Cas9 is a recently reported Cas9 homolog that has a dinucleotide $\left(\mathrm{N}_{4} \mathrm{CC}\right.$ ) protospacer adjacent motif (PAM) (Edraki et al. 2018), enabling a target site density comparable to that of SpyCas9 (NGG PAM). A Type II-C Nme1Cas9 or Nme2Cas9 inhibitor, AcrllC3 ${ }_{\text {Nme }}$ (Pawluk et al. 2016), staples two Cas9s together and prevents DNA cleavage (Harrington et al. 2017; Zhu et al. 2019). AcrllA4 Lmo $_{\text {inhibits }}$ the widely used SpyCas9 and blocks DNA binding, in this case by occluding the PAM-binding cleft (Dong et al. 2017; Rauch et al. 2017; Shin et al. 2017; Yang and Patel

B poly(A) tail.
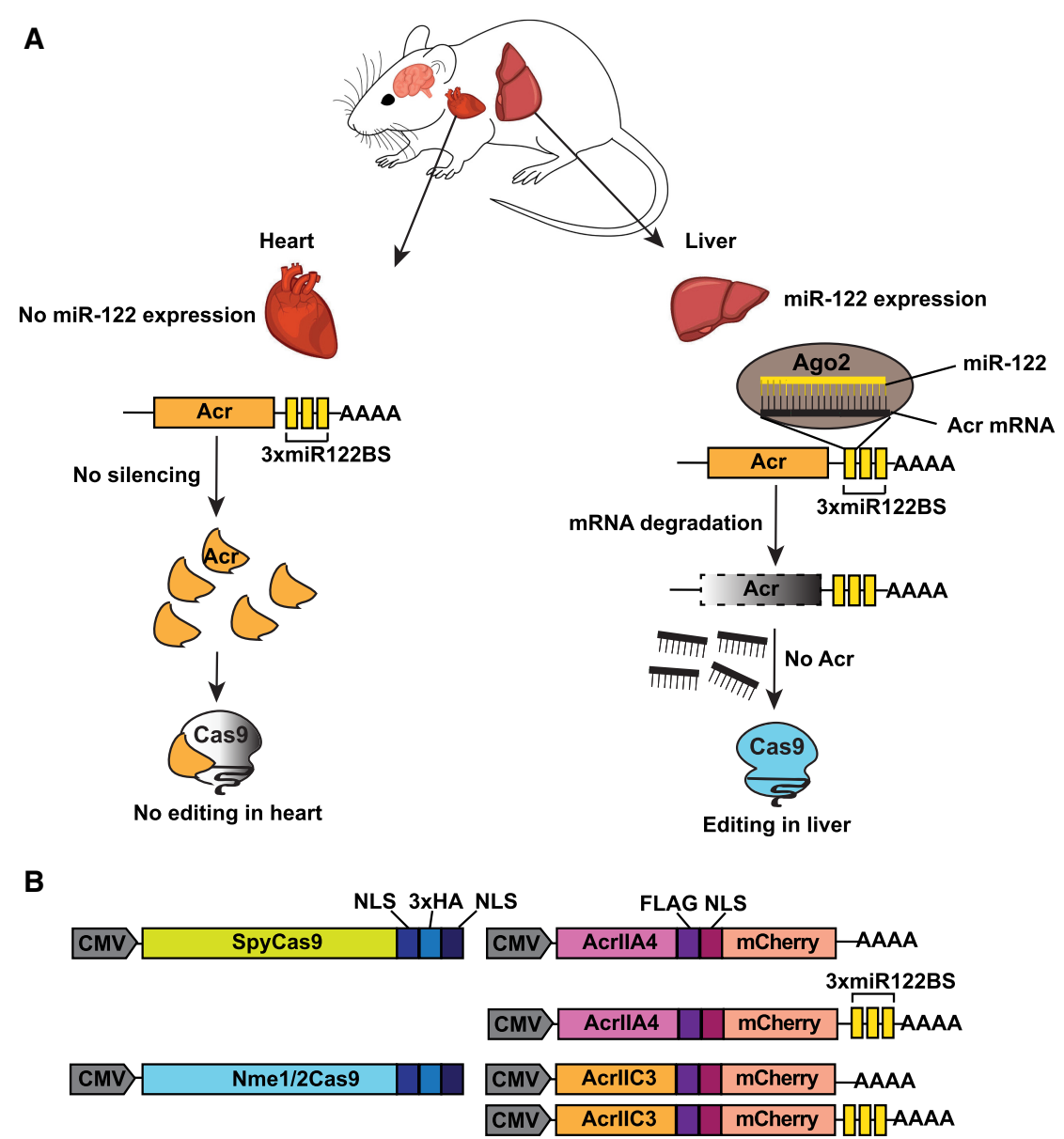

FIGURE 1. Overview of the Cas9 and microRNA-repressible anti-CRISPR system. (A) Outline of the miRNA-repressible anti-CRISPR and Cas9 editing strategy as designed for use in mice. As an example, miR-122 can be used to achieve liver-specific editing. Upon systemic delivery of Cas9 in vivo (e.g., via viral vectors), tissues receiving Cas9 and sgRNA potentially result in genome editing; however, codelivery of miRNA-repressible anti-CRISPR proteins will prevent such editing in nontarget tissues that lack miR-122, as depicted in the heart (left). In the liver, anti-CRISPR transcripts with perfectly complementary miR-122 binding sites will undergo Ago2-mediated mRNA degradation, and the resulting silencing of the Acr will permit Cas9 editing in the liver (right). (B) A schematic of expression vectors for Cas9 orthologs from Type II-A (SpyCas9) and II-C (Nme1Cas9 and Nme2Cas9) systems, along with their respective anti-CRISPR proteins, AcrllA4 and AcrlIC3. The Acr expression constructions were generated with or without three tandem, perfectly complementary miRNA-122 binding sites in the $3^{\prime}$ UTR. (CMV) cytomegalovirus promoter, (NLS) nuclear localization signal, (AAAA),

2017). For our in vitro validations, both Cas 9 and Acr expression vectors were driven by the cytomegalovirus (CMV) promoter. We generated codon-optimized Acr expression vectors identical in every respect except for the presence or absence of MREs in the $3^{\prime}$ UTR (Supplemental Table S1). Since miR-122 is a well-validated miRNA that is highly expressed specifically in hepatic cells, we decided to test this concept using this miRNA. We placed three tandem miR-122 binding sites (3xmiR122BS) in the $3^{\prime}$ UTR of each Acr gene, which also included a carboxy-terminal mCherry fusion to enable 
expression to be detected by fluorescence microscopy or flow cytometry (Fig. 1B). The fusion of heterologous domains do not compromise the inhibitory potency of these Acrs (Basgall et al. 2018; Nakamura et al. 2019).

\section{Validation of microRNA- repressible anti-CRISPR expression vectors}

We used a human hepatocellular carcinoma cell line (Huh-7) that abundantly expresses miR-122, in contrast to nonhepatic cell lines such as human embryonic kidney (HEK293T) cells (Fukuhara et al. 2012). As an initial test of miR-122 repression of Acr expression, we transfected cells with plasmids expressing AcrllC3Flag-mCherry-3xmiR122BS, AcrllA4Flag-mCherry-3xmiR122BS, or their respective control vectors lacking the miR-122 binding sites (Fig. 1B). A separate GFP expression plasmid was also included to indicate transfection efficiencies in each cell line. When these vectors were transiently transfected, the expression of mCherry-fused Acr with miR-122 MREs was dramatically suppressed in Huh7 cells whereas Acr-mCherry lacking the 3xmiR122BS cassette was still well expressed (Fig. 2A). In HEK293T cells, there was no discernible difference in mCherry signal from the Acr and Acr-3xmiR122BS constructs based on both fluorescence microscopy and flow cytometry (Fig. 2B). Acr expression was also confirmed by anti-Flag western blot analysis (Fig. 2). Compared to HEK293T cells, transfection efficiency was lower in Huh-7 cells as indicated by a decrease in overall GFP and mCherry signals (Fig. 2A). Nevertheless, fluorescence microscopy, flow cytometry, and western blot analysis consistently revealed effective reductions of both AcrllC3-3xmiR122BS and AcrllA4-3xmiR122BS expression in Huh-7, but not in HEK293T cells. Expression of Acrs lacking miR-122 MREs was unaffected in both cell lines, consistent with effective regulation of Acr by miR-122 only in hepatic cells.

B Scale bar, $100 \mu \mathrm{m}$.
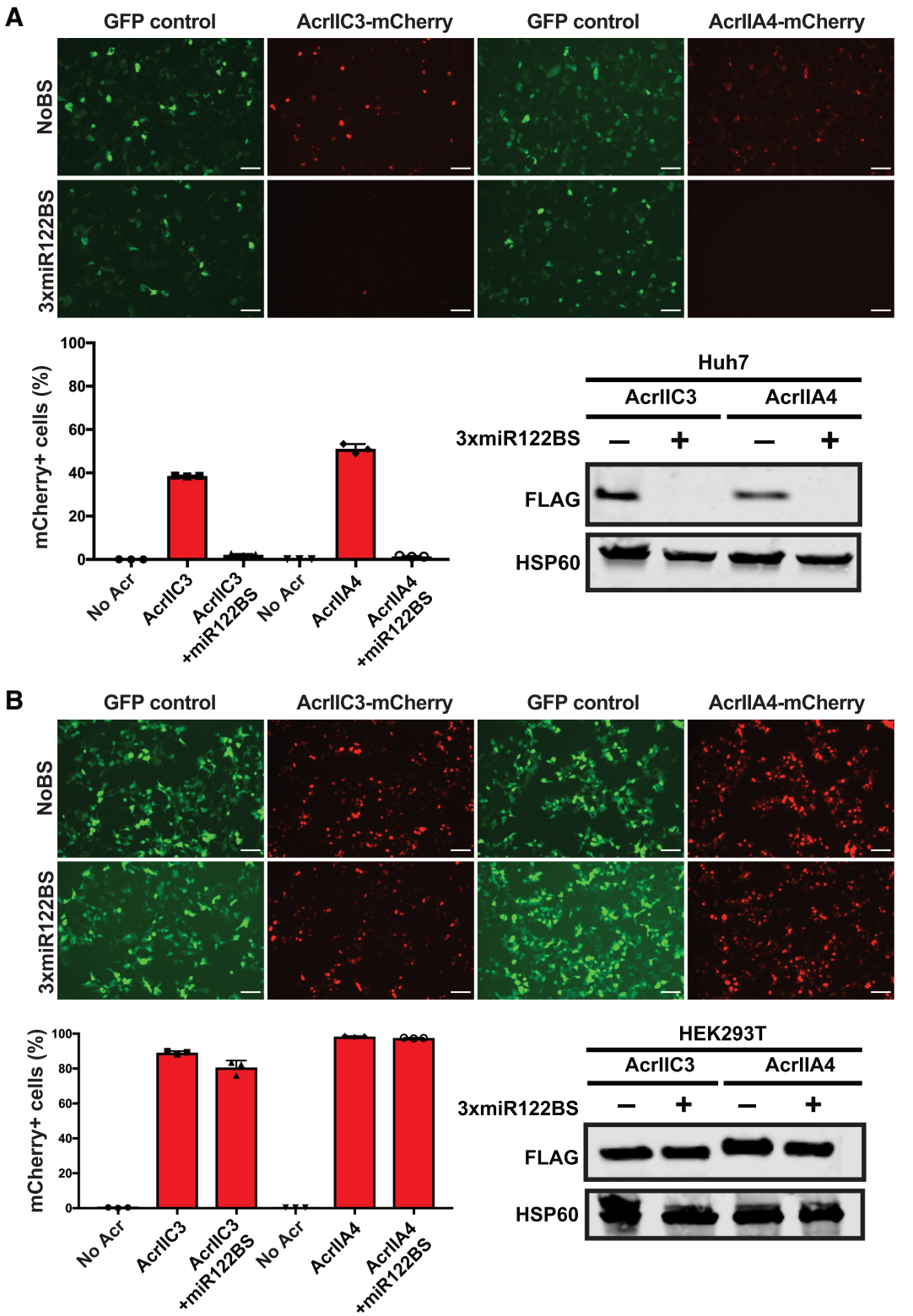

FIGURE 2. Validation of miRNA regulation of anti-CRISPR expression in cultured cells. $(A, B)$ Hepatocyte-specific silencing of anti-CRISPR expression. Plasmid vectors shown in Figure 1B encoding either AcrllC3-mCherry or AcrllA4-mCherry, with or without miR-122 MREs, were transfected into (A) human hepatoma (Huh7) cells or (B) nonhepatic HEK293T cells; only the former express miR-122. The expression of $m$ Cherry and GFP was visualized by fluorescence microscopy (top) and analyzed by flow cytometry (bottom left). The percentage of mCherrypositive cells in each transfection was normalized to transfection of the control GFP-expressing plasmid. Anti-CRISPR protein expression was also confirmed by western blot against the 1xFlag epitope (bottom right). Heat shock protein 60 (HSP60) was used as a loading control.

\section{MicroRNA repression enables escape from anti- CRISPR inhibition during genome editing in hepatocyte-derived cells}

Having demonstrated that anti-CRISPR repression in hepatocyte-derived cells can be conferred by miR-122 MREs, 
we then tested whether this repression is sufficient to allow genome editing by Cas9 orthologs (SpyCas9, Nme1Cas9, and $\mathrm{Nme2Cas9).} \mathrm{We} \mathrm{transiently} \mathrm{transfected} \mathrm{separate} \mathrm{ex-}$ pression plasmids for Cas9, a cognate sgRNA, and an $A c r$, with the latter construct either including or omitting miR-122 binding sites. We chose validated, endogenous sites in the human genome for each Cas9 ortholog

A

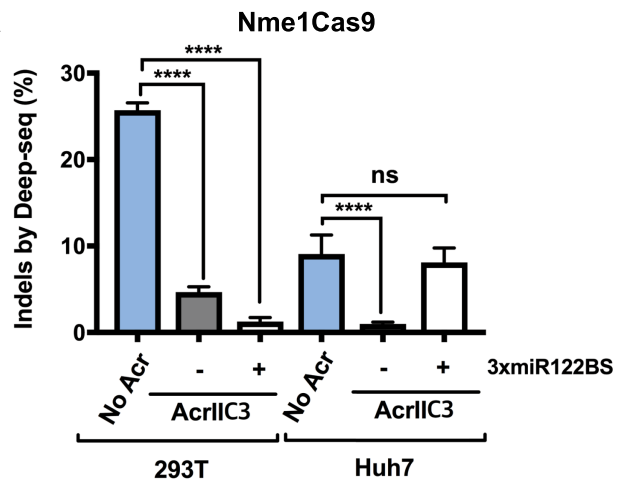

B

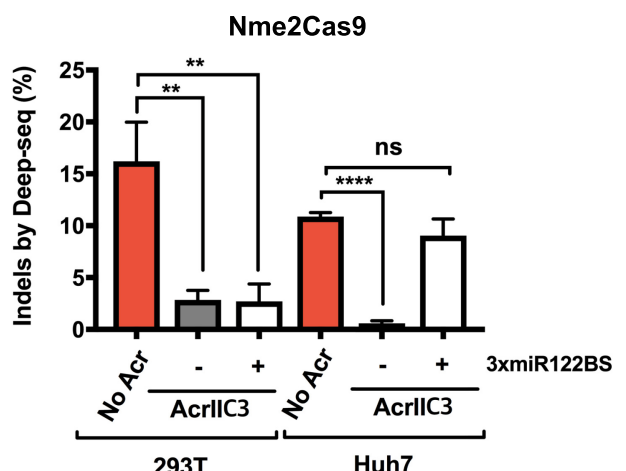

C

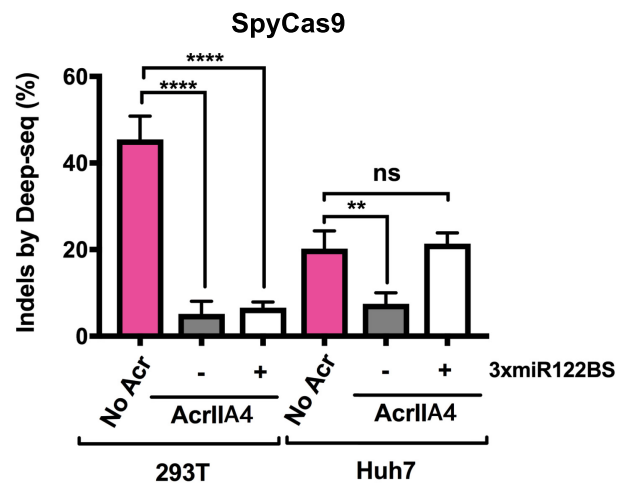

FIGURE 3. Hepatocyte-specific genome editing by Nme1Cas9, Nme2Cas9, and SpyCas9 in cultured cells. (A-C) HEK293T and Huh7 cells were transiently transfected with plasmids encoding $(A)$ Nme1Cas9 and a sgRNA targeting the VEGFA locus, (B) Nme2Cas9 and a sgRNA targeting LINC01588, and (C) SpyCas9 and a sgRNA targeting the VEGFA locus. $(A, B)$ AcrllC3 constructs with or without $3 \times$ miR122BS were cotransfected with the Cas9 and sgRNA constructs as indicated. (C) AcrllA4 with or without $3 \times$ miR122BS were cotransfected with SpyCas9 and its sgRNA. Data represent mean \pm SD with at least three replicates. Editing efficiencies are measured by targeted deep sequencing.
(Fig. 3): the Nme1Cas9 target site NTS33 in the VEGFA gene (Fig. 3A), the Nme2Cas9 target site TS6 in the LINC01588 gene (Fig. 3B), and the SpyCas9 target site STS3 in the VEGFA gene (Fig. 3C; Amrani et al. 2018; Edraki et al. 2018). In HEK293T cells, AcrllC3 and AcrllA4 robustly inhibited genome editing by $\mathrm{Nme1/}$ 2Cas9 and SpyCas9, respectively, as expected (Fig. 3; Pawluk et al. 2016; Rauch et al. 2017). The presence or absence of miR-122 MREs had no significant effect on editing inhibition in this non-miR-122-expressing cell type. Although the editing efficiency was variable among Cas9 orthologs at these target sites, and although transfection efficiencies were reduced in Huh-7 cells, AcrllC3 and AcrllA4 also prevented editing in this cell type when expressed from constructs that lack miR-122 MREs. In contrast, Acr plasmids that incorporated miR-122 MREs in the $3^{\prime}$ UTRs failed to inhibit Cas9 editing in Huh-7 cells, as indicated by editing efficiencies that were similar to the no-Acr control (Fig. 3). This trend was true for all three Cas9 orthologs tested.

\section{MiR-122-dependent genome editing conferred by an anti-CRISPR protein in vivo}

For our in vivo tests, we focused on Nme2Cas9, due to its compact size, high target site density, and relative lack of off-target editing (Edraki et al. 2018), all of which are advantageous for therapeutic development. We used a previously validated all-in-one AAV vector that expresses Nme2Cas9 from the minimal U1a promoter, as well as a U6 promoter-driven sgRNA targeting Rosa26 (Fig. 4A; Edraki et al. 2018; Ibraheim et al. 2018). We also generated AcrllC3 expression plasmids driven by the strong CB-PI promoter and associated expression elements; in addition, these AcrllC3 constructs either included or omitted the three tandem miR-122 MREs in the 3'-UTR (Fig. 4A). For in vivo delivery we first used hydrodynamic injection, which is a nonviral method of transient hepatocyte transfection that allows expression from naked DNA plasmids (Zhang et al. 1999). This injection method delivers DNA to $10 \%-20 \%$ of hepatocytes for transient expression and leads to minimal transgene expression in organs other than the liver. Since miR-122 is abundant in the liver, and because Cas 9 delivered to the liver by hydrodynamic injection can induce editing (Xue et al. 2014), this experimental approach enables tests of liver-specific editing (and inhibition of editing) in the presence or absence of Acr expression. Plasmids were injected into adult, wildtype C57BL/6 mice via tail vein and liver tissues were collected at $7 \mathrm{~d}$ post-injection (Fig. 4B). To determine the effective dose of Acr plasmid needed to inhibit Nme2Cas9 editing in vivo, we coinjected varying Cas9: Acr plasmid ratios (1:1, 1:1.5, and 1:2). AcrllC3 efficiently inhibited Nme2Cas9 editing at all ratios tested (Fig. 4C). No apparent liver damage was detected in the liver tissues 


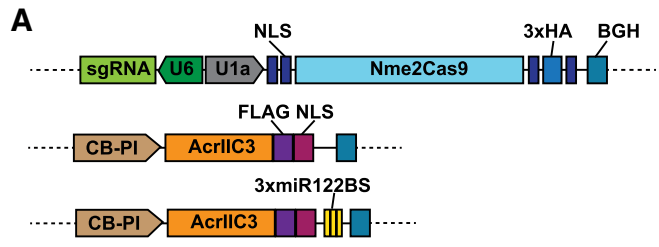

B

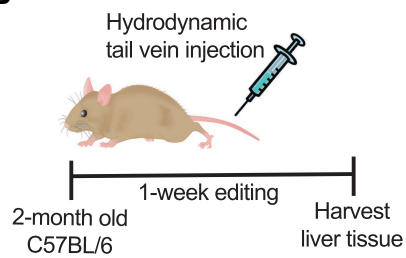

C

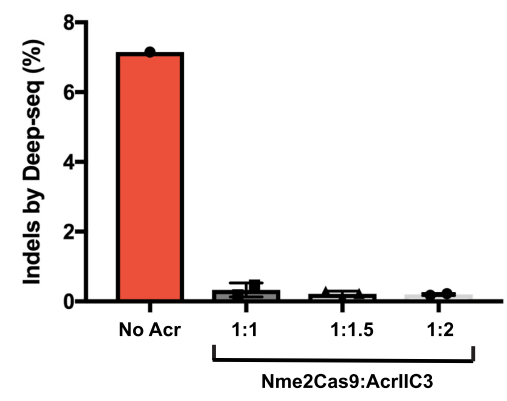

D

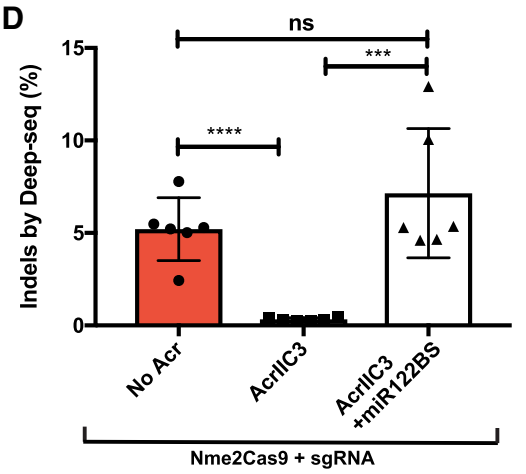

FIGURE 4. Acr inhibition of Nme2Cas9 editing in vivo, and release from inhibition by the liverspecific miRNA, miR-122. (A) Plasmids used for in vivo studies to drive the expression of Nme2Cas9 + sgRNA and AcrllC3, respectively. (U1a) murine promoter, (BGH) bovine growth hormone poly(A) signal, (CB-PI), cytomegalovirus-enhancer, chicken $\beta$-actin (CB) promoter with SV40-derived mini-intron. (B) A schematic of mouse tail vein injection studies. Plasmid vectors shown in $(A)$ are administered into 8- to 10-wk-old C56BL/6 mice by hydrodynamic injection. Liver tissues were collected 1 wk after injection. (C) Dose titration of Nme2Cas9+ sgRNA plasmid to AcrllC3 plasmid in vivo. Percentage of indels at the Rosa26 target in the livers of C57Bl/6 mice were measured by targeted deep sequencing after hydrodynamic injection of Nme2Cas9 + sgRNA and AcrllC3 plasmids at mass ratios of 1:1, 1:1.5, and 1:2. (D) Genome editing in the liver by Nme2Cas9 is inhibited by AcrlIC3 but restored when AcrllC3-3xmiR122BS is silenced. Indel percentages at the Rosa26 locus in the livers of C57BI/6 mice were measured by targeted deep sequencing after hydrodynamic injection of Nme2Cas9 + sgRNA plasmid, along with AcrllC3 plasmids with or without 3xmiR122BS. $n=$ 6 mice per group. ns = not significant, $P<0.05$ by unpaired, two-tailed t-test.

following staining with haemotoxylin and eosin (H\&E) (Supplemental Fig. S2A). Once we defined the necessary plasmid dose, we subjected three groups of mice to hydrodynamic injection with plasmid combinations that included Nme2Cas9 with (i) no Acr, (ii) AcrllC3, and (iii) AcrllC3-3xmiR122BS (Fig. 4A). In the livers of mice receiving no Acr, Nme2Cas9 yielded a mean editing efficiency of $5.2 \pm 1.7 \%$ ( $n=6$ mice), similar to levels seen previously with this and other Cas9 orthologs upon hydrodynamic injection (Xue et al. 2014; lbraheim et al. 2018). As expected, coinjection of AcrllC3 plasmid strongly reduced the editing efficiency to $0.33 \pm 0.09 \%(P<0.0001)$. In contrast, AcrllC3-3xmiR122BS failed to inhibit Nme2Cas9 editing, with the indel efficiency comparable to no Acr group $(7.1 \pm 3.5 \%$, Fig. 4D). We confirmed the expression of Nme2Cas9 in all three groups by immunohistochemistry (IHC) against the 3xHA epitope (Supplemental Fig. S2B). We were unable to detect AcrlIC3 by IHC against the Flag epitope in mice injected with AcrllC3, likely because antibody binding by the $1 \times$ Flag tag is too weak for $\mathrm{IHC}$ detection under these conditions. However, we ruled out the possibility of injection failures by including control plasmids in our experiment. Specifically, we coinjected additional plasmids encoding a Sleeping Beauty transposon system (Ivics et al. 1997) that integrates a mCherry expression cassette into the mouse genome to report on the success of plasmid injection. In all three groups of injected mice, we observed mCherry expression in liver tissue sections from injected mice by $\mathrm{IHC}$ (Supplemental Fig. S2B), confirming successful hepatocyte transfection. In addition, we used RT-PCR to confirm mRNA expression in the liver tissues of mice injected with the indicated Nme2Cas9 and AcrllC3 vectors, but not in PBS-injected mice (Supplemental Fig. S2C). In summary, consistent with our results in human Huh-7 cells, endogenous miR-122 in mouse hepatocytes in vivo can be exploited to repress Acr expression, and therefore allow tissue-specific Cas9 genome editing, in liver.

\section{Systemic AAV delivery of a miRNA-repressible anti-CRISPR inhibits off-tissue genome editing}

To demonstrate that the miRNArepressible anti-CRISPR proteins can inhibit editing in nontarget tissue upon systemic delivery, we designed a dual-AAV system in which a single-stranded AAV (sSAAV) vector expressing Nme2Cas9 is codelivered with a self-complementary AAV (scAAV) expressing an anti-CRISPR protein and a cognate sgRNA (Fig. 5A). We used scAAV for AcrllC3 expression to enable the earlier onset of transcription (before second-strand synthesis). This expedited AcrllC3 expression maximizes the likelihood that inhibitory levels of the anti-CRISPR can accumulate before significant sSAAVbased Nme2Cas 9 expression occurs, since the latter requires prior synthesis of the complementary vector strand. Furthermore, we transferred the U6-driven sgRNA cassette from our previously developed all-in-one ssAAVNme2Cas9 vector to the Acr-expressing scAAV vector to ensure that editing cannot occur in cells that fail to receive the Acr transgene. These vectors were packaged as serotype nine capsids (AAV9), which are known to have a particularly broad tissue tropism in mice (Zincarelli et al. 2008). The tail veins of three groups of mice ( $n=6$ for each group) were injected with $4 \times 10^{11}$ genome copies (GC) of ssAAV9-Nme2Cas9 vector, along with $4 \times 10^{11}$ 
A

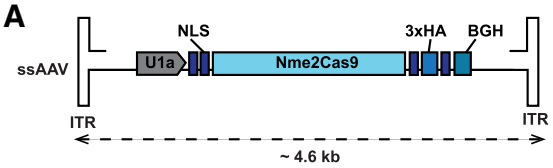

B

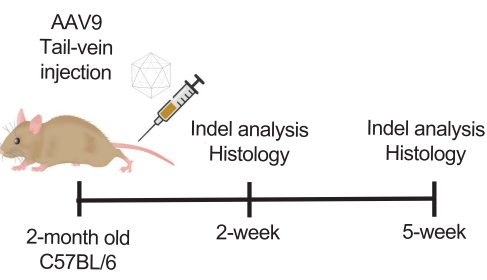

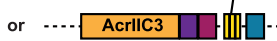

or $\cdots . .$. AcrllA4

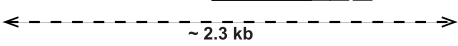

C

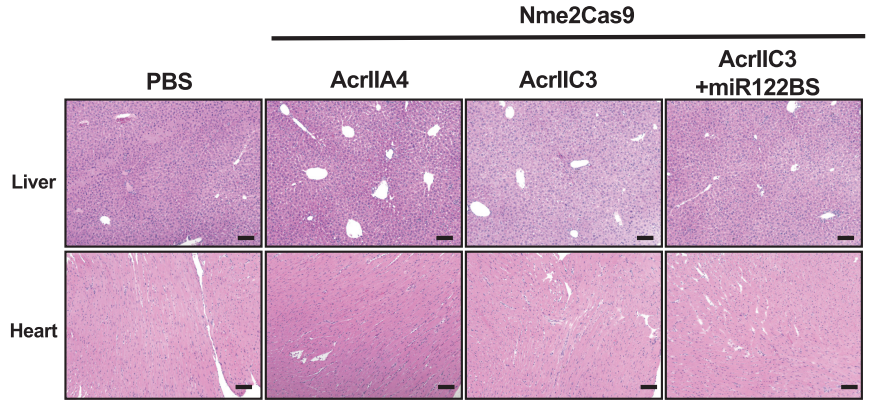

D
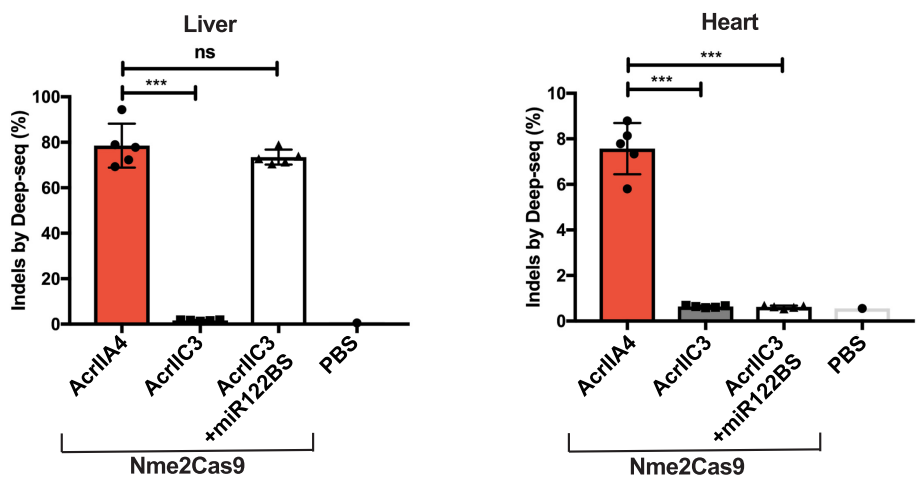

FIGURE 5. AAV delivery of a miRNA-repressible AcrllC3 inhibits Nme2Cas9 editing in a nontarget tissue. (A) Design of a dual-AAV9 system for Nme2Cas9, sgRNA, and anti-CRISPR expression. A single-stranded AAV9 (ssAAV9) vector encodes Nme2Cas9 and a self-complementary AAV9 (scAAV9) vector encodes an anti-CRISPR protein (AcrllC3, AcrllC33xmiR122BS, or AcrllA4) as well as a U6-driven sgRNA targeting Rosa26. (U1a) murine promoter, (BGH) bovine growth hormone poly(A) signal, (CB-PI) cytomegalovirus-enhancer, (CB) chicken $\beta$-actin promoter with SV40-derived mini-intron, (ITR) inverted terminal repeat. (B) A schematic of mouse studies for AAV9 delivery. The dual-AAV9 system shown in (A) was administered into 8- to 10-wk-old C56BL/6 mice via tail vein injection. Liver and heart tissue samples were collected at 2- and 5-wk post-injection. (C) H\&E staining of liver and heart tissue sections collected from mice $5 \mathrm{wk}$ after AAV9 injection. Scale bar, $100 \mu \mathrm{m}$. (D) Genome editing in liver and heart tissue samples 5 wk after AAV delivery. Nme2Cas9 is inhibited by AcrllC3 in both liver and heart tissues. In contrast, when AcrllC3-3xmiR122BS is used, editing is restored in the liver but not in the heart, due to anti-CRISPR repression by the hepatocyte-specific miR122. Indel percentages at the Rosa26 locus were measured by targeted deep sequencing For the 5 -wk time points, $n=5$ mice per group. $n s=$ not significant; $P<0.05$ by unpaired, two-tailed t-test. Control, PBS-injected.

GC of either (i) scAAV9-AcrllC3-sgRNA, (ii) scAAV9AcrllC3-3xmiR122BS-sgRNA, or (iii) scAAV9-AcrllA4sgRNA vector (Fig. 5B). AcrllA4 was used as a noncognate, negative-control anti-CRISPR (Fig. 5A). Both liver and heart tissue samples were collected for indel analysis and histol- ogy at 2-wk $(n=1$, Supplemental Fig. S4) and 5-wk $(n=5)$ time points (Fig. 5). We observed no apparent tissue damage associated with AAV9 delivery of Nme2Cas9 and anti-CRISPR proteins following H\&E staining of liver and cardiac muscles (Fig. 5C). Consistent with in vivo delivery by hydrodynamic injection, editing in liver was inhibited by AcrllC3 but not by AcrllC3-3xmiR122BS, in accord with microRNA silencing of the latter in hepatocytes (Fig. 5D). In contrast, off-tissue editing in the heart was inhibited by both of the AcrllC3 and AcrllC3-3xmiR122BS vectors, indicating that the latter was effectively expressed in the absence of miR-122 in cardiomyocytes. This trend was observed in tissues collected at both 2- and 5-wk time points, although (as expected) editing efficiencies were higher overall at $5 \mathrm{wk}$ post-injection (Supplemental Fig. S3A). Robust Nme2Cas9 expression was detected by $\mathrm{IHC}$ in the liver as well as the cardiac muscles at both time points, corroborating that the lack of editing was indeed due to AcrllC3 inhibition and not due to lack of Nme2Cas9 expression (Supplemental Figs. S3B, S4A). We also used RT-PCR to confirm that AcrllC3 mRNA is expressed in these tissues (Supplemental Fig. S4B). Collectively these results demonstrate that Type II anti-CRISPRs can be used as AAV-deliverable offswitches for genome editing in vivo, and furthermore that they can be effectively rendered miRNA-repressible to enforce the tissue specificity of genome editing activity.

\section{DISCUSSION}

Although CRISPR-Cas9 technologies have immense promise in numerous aspects of biomedical science, many applications will benefit from tight temporal or spatial control over Cas9 activity, especially in the context of clinical development. Confining Cas9 activity to target cells and tissues of interest is highly desirable to prevent unforeseen adverse effects associated with off-tissue and off-target editing in vivo. Natural inhibitors of Cas proteins, anti-CRISPRs, 
can be repurposed as tools to limit the potential for unwanted edits. Acrs have several potential advantages for implementation as regulators. They are natural and genetically encodable inhibitors of Cas nucleases that have evolved as powerful inactivators of CRISPR immunity, usually offering some degree of specificity for particular types of CRISPR-Cas systems. Moreover, their inhibition is often tunable or titratable based on the relative expression levels of Acrs and the target effectors, given the stoichiometric mechanisms of action for most of them (Bondy-Denomy 2018; van Gent and Gack 2018). Most Acrs are small proteins that can tolerate fusions of fluorescent proteins or epitope tags, which could make them convenient for in vivo delivery by viral vectors or mRNAs and detection by fluorescence.

Here we present a proof-of-concept demonstration of anti-CRISPR regulation by endogenous miRNAs in vivo, yielding tissue-specific control over CRISPR-Cas9 editing. In preliminary work, we demonstrated that miRNA-mediated inhibition of anti-CRISPRs bearing hepatocyte-specific miR-122 MREs allows genome editing in a human hepatocyte cell line, but inhibits editing in a nonhepatocytederived cell line. Although this study used AcrllC3 for Type II-C Nme1Cas9 and Nme2Cas9, as well as AcrllA4 for Type II-A SpyCas9, any well-validated combination of Acr-Cas nuclease will be compatible with this strategy, making it a versatile platform. With the wealth of new Acrs emerging for different CRISPR effectors (e.g., Cas12a; (Marino et al. 2018; Watters et al. 2018), we expect that opportunities for implementing this strategy will continue to increase. Expression profiles of many miRNAs are well-defined for many tissues at many developmental stages and in numerous disease states (Alvarez-Garcia and Miska 2005). For example, miR-1 is highly and specifically expressed in cardiac and skeletal muscle tissues (Horak et al. 2016). The miRNA-repressible Acr system affords great flexibility in changing editing tissue specificity, given the ease with which the MREs can be swapped into the 3'UTR of the Acr transcript. Furthermore, because MREs are so small, this approach is well suited for viral modes of delivery (given the genome capacity constraints of viral vectors), and could confer specificity for some tissues that lack vector-compatible, tissue-specific promoters.

We extend this strategy to animal studies that document anti-CRISPR efficacy during Cas9-mediated editing in vivo in adult mammals. To our knowledge, this is the first demonstration of in vivo expression of Acr proteins in vertebrate models to inhibit Cas9 editing activity. From this study, we did not observe overt toxicity in the examined tissues, although the safety and immunity profiles of delivered Acr proteins will need to be investigated over longer periods of time and in additional biological contexts. Importantly, we exploited endogenous miRNAs for spatial control of anti-CRISPR expression to achieve tissue-specif- ic editing by Nme2Cas9 in vivo in adult mice. The endogenous miRNA repertoire has been combined with the CRISPR-Cas machinery previously to regulate the expression of Cas9 itself (Senís et al. 2014; Hirosawa et al. 2017). Whereas detargeting Cas9 expression from the liver (e.g. with miR-122) will allow editing to occur everywhere except the liver, our strategy will instead restrict Cas9 activity to the liver itself and protect other tissues from unwanted editing events. This will be particularly useful to restrict Cas9 genome editing to a single desired tissue following a systemic Cas9 delivery by AAV. Our results complement a strategy that exploits miRNAs to release sgRNAs from longer, inactive precursors (Wang et al. 2019), though this approach has not yet been validated in tissue-specific editing applications in vivo. While our manuscript was in preparation and revision, two reports also described miRNA-regulated Acr strategies that enable cell-type specific editing in cultured hepatocytes and myocytes (Hirosawa et al. 2019; Hoffmann et al. 2019). Our studies extend this work by establishing that miRNA-repressible anti-CRISPRs can enforce the tissue specificity of genome editing in discrete organs of adult mammals in vivo.

\section{MATERIALS AND METHODS}

\section{Vector construction}

Codon-optimized AcrllC3 and AcrllA4 sequences were ordered as gBlocks (IDT) and amplified using the primers with overhangs to the pCSDest vector by NEBuilder HiFi DNA Assembly (NEB). Similarly, an mCherry ORF was fused to the caboxyl terminus of each Acr by HiFi DNA assembly (NEB). To insert 3xmiR122 MREs in the 3' UTR of each Acr, top and bottom strands were ordered as oligos (IDT) with restriction sites for Sacl and HindIII and annealed before ligating into the vector linearized with the same restriction enzymes. For in vivo experiments involving a hydrodynamic injection, we used the Nme2Cas9-sgRNA_Rosa26 allin-one AAV vector (Edraki et al. 2018). To make scAAV vectors expressing Acr proteins, the original scAAV plasmid encoding an EGFP ORF [a kind gift from Jun Xie and Guangping Gao (UMass Medical School)] and pCSDest-Acr plasmids were digested with Sacl and Agel restriction enzymes and then ligated. For AAV vector preparation, a U6-driven sgRNA cassette was removed from the Nme2Cas9 vector by restriction digestion with Mlul and assembled into linearized scAAV-EGFP by Hifi DNA assembly (NEB). This vector was digested with Sacl and Agel for AcrllC3, AcrllC3-3xmiR122BS, or AcrllA4 inserts made from pCSDest plasmids using the same restriction enzymes. The sequences of codon-optimized Acr constructs and miRNA-122 MREs are provided in the Supplemental Table S1. All plasmids used in this study are summarized in Supplemental Table S2 and will be made available from Addgene.

\section{Cell culture and transfection}

HEK293T and Huh-7 cell lines were cultured in Dulbecco's modified Eagle's medium supplemented with $10 \%$ fetal bovine serum 
(Sigma) and 1\% penicillin-streptomycin (Gibco). For editing experiments in vitro, a total of $150 \mathrm{ng}$ of Cas9, $150 \mathrm{ng}$ of sgRNA, and $50 \mathrm{ng}$ of Acr or an empty plasmid were transiently transfected in a 24-well format using Lipofectamine 2000 (Invitrogen) according to the manufacturer's protocol. The total DNA amount was kept constant by adding a stuffer plasmid in all cases. For western blot and flow cytometry analysis, 500,000 cells/well were seeded onto a six-well plate, and $500 \mathrm{ng}$ of each Acr vector and GFP plasmid (the latter used as a transfection control) were transfected using Lipofectamine 2000 (Invitrogen) following the manufacturer's protocol. Prior to flow cytometry analysis, cells were imaged using an EVOS Cell Imaging System (Thermo Fisher).

\section{Flow cytometry}

Cells were trypsinized $48 \mathrm{~h}$ post-transfection, washed with PBS, and resuspended in PBS. A total of 100,000 cells were analyzed on a MACSQuant ${ }^{\circledR}$ VYB (Miltenyi Biotec). A yellow laser (561 $\mathrm{nm}$ ) with a $615 / 20 \mathrm{~nm}$ filter and a blue laser $(488 \mathrm{~nm})$ with a 525/50 nm filter were used for mCherry and GFP detection, respectively. Subsequent analysis was performed using FlowJo v10.4.1. Cells were first sorted based on forward and side scattering (FSC-A vs. SSC-A), and then single cells were gated using FSC-A and FSC-H. Finally, mCherry-positive cells were recorded to estimate the expression level of anti-CRISPR proteins after gating for GFP-positive (transfected) cells.

\section{Western blots}

Proteins were collected $48 \mathrm{~h}$ post-transfection and their concentrations were measured using the Pierce BCA Protein Assay Kit (Thermo Fisher Scientific). Western blots were performed as described previously (Lee et al. 2018) with primary mouse antiFlag (AbClonal, 1:5000) used for Acr detection and rabbit antiHSP60 (1:5000) used for loading control. After incubation with secondary anti-Rabbit or anti-Mouse antibodies (LI-COR IRDye, 1:20,000), blots were visualized using a LI-COR imaging system.

\section{Mouse studies}

C57BL/6 mice were obtained from Jackson Laboratory and all animal maintenance and procedures were performed following the guidelines of the Institutional Animal Care and Use Committee of the University of Massachusetts Medical School. Plasmids for hydrodynamic tail vein injection were prepared using the EndoFreeMaxi kit (Qiagen). For hydrodynamic liver injection, a total of $90 \mu \mathrm{g}$ of endotoxin-free plasmids was suspended in 2 $\mathrm{mL}$ of injection-grade saline and injected via the tail vein into 8- to 10-wk-old C57BL/6 mice. Mice were euthanized $7 \mathrm{~d}$ postinjection and liver tissues were collected and stored at $-80^{\circ} \mathrm{C}$ for analyses. For AAV injection, $4 \times 10^{11}$ GC of ssAAVNme2Cas9 and $4 \times 10^{11} \mathrm{GC}$ of scAAV-U6_sgRNA-Acr (a total of $8 \times 10^{11}$ GC per mouse) were resuspended in $200 \mu \mathrm{L}$ PBS and administered via tail vein injection. Tissue samples from heart and liver were collected and stored at $-80^{\circ} \mathrm{C}$ for indel analysis and histology at 2- and 5-wk post-injection.

\section{Indel analysis}

Genomic DNA from cells or tissues were collected using a DNeasy Blood and Tissue Kit (Qiagen). Indel frequencies were measured by targeted deep sequencing. Targeted deep sequencing analyses were done as previously described (Bolukbasi et al. 2015). Briefly, target sites were amplified using High Fidelity 2x PCR Master Mix (NEB) in a two-step PCR amplification with locus-specific primers in the first step and then with universal index primers to reconstitute TruSeq adapters. Fullsize products were gel-extracted and purified using a DNA Clean and Concentrator Kit (Zymo). The purified library was sequenced using a paired-end $150 \mathrm{bp}$ MiniSeq run using a Midoutput cartridge (Illumina). Raw deep sequencing data are provided in the Supplemental Excel File.

\section{Immunohistochemistry}

Liver tissues were fixed in $4 \%$ formalin overnight, paraffinembedded, and sectioned at the UMass Morphology Core. For Figure $5 \mathrm{C}$ and Supplemental Figure S2A, sectioned slides were stained with H\&E for pathology analysis. For IHC, liver sections were dewaxed, rehydrated, and stained following standard protocols previously described (Xue et al. 2011) with primary antibodies against 3xHA-tagged Nme2Cas9 (anti-HA; Cell Signaling) and mCherry (anti-RFP; Rockland). Representative images are shown.

\section{RT-PCR analysis}

Total RNA $(0.5-1 \mu \mathrm{g})$ from mouse tissues were collected by TRIzol (Invitrogen) and then reverse-transcribed to cDNA using random hexamer primers and SuperScript III First-Strand Synthesis System (Invitrogen) following the manufacturers' protocols. cDNA templates were directly used for PCR amplification using primers specific for (1) Nme2Cas9, (2) AcrllC3, and (3) GAPDH. Primer sequences are provided in Supplemental Table S2. The resulting RT-PCR amplicons were visualized by $2.5 \%$ agarose $/ 1 \times$ TAE gel electrophoresis.

\section{Statistical analysis}

Standard deviations are derived from each group that has a minimum of three independent replicates unless otherwise noted. Unpaired, two-tailed t-tests were used to determine the statistical significance between each group. Resulting $P$ values $<0.05,0.01$, $0.001,0.0001$ are indicated by one, two, three, or four asterisks, respectively.

\section{DATA DEPOSITION}

High-throughput sequencing data is available in the $\mathrm{NCBI}$ Sequence Read Archive (SRA) under the BioProject accession code PRJNA555639. All other relevant data are available from the corresponding authors upon request.

\section{SUPPLEMENTAL MATERIAL}

Supplemental material is available for this article. 


\section{COMPETING INTEREST STATEMENT}

E.J.S. is a cofounder and scientific advisor of Intellia Therapeutics.

\section{ACKNOWLEDGMENTS}

We thank Jun Xie, Guangping Gao, and members of the Xue and Sontheimer labs for helpful discussions and sharing resources. We also thank Jordan Smith for assistance with IHC experiments, as well as Kevin Luk, Pengpeng Liu, and Scot Wolfe for sharing sgRNA plasmids. We thank the UMMS Viral Vector Core for AAV production and the Department of Animal Medicine for mouse injections with AAV vectors. This work was supported by grants from the U.S. National Institutes of Health (GM125797) to E.J.S. and (DP2HL137167 and UG3HL147367) to W.X. as well as institutional funds to W.X. and E.J.S.

Author contributions: J.L. constructed all new plasmids used in this study, conducted all cell culture experiments, and analyzed samples derived from in vivo experiments. H.M. and S.-Q.L. performed hydrodynamic injection and mouse tissue collection with guidance from W.X. R.I. provided tissue samples from mice injected with AAV8. P.L. analyzed the deep sequencing data. J.L. and E.J.S. wrote the manuscript and all authors edited the manuscript.

Received May 1, 2019; accepted August 9, 2019.

\section{REFERENCES}

Alvarez-Garcia I, Miska EA. 2005. MicroRNA functions in animal development and human disease. Development 132: 4653-4662. doi:10.1242/dev.02073

Amrani N, Gao XD, Liu P, Edraki A, Mir A, Ibraheim R, Gupta A, Sasaki KE, Wu T, Donohoue PD, et al. 2018. NmeCas9 is an intrinsically high-fidelity genome editing platform. Genome Biol 19: 214. doi:10.1186/s13059-018-1591-1

Bartel DP. 2018. Metazoan microRNAs. Cell 173: 20-51. doi:10.1016/ j.cell.2018.03.006

Basgall EM, Goetting SC, Goeckel ME, Giersch RM, Roggenkamp E, Schrock MN, Halloran M, Finnigan GC. 2018. Gene drive inhibition by the anti-CRISPR proteins AcrllA2 and AcrllA4 in Saccharomyces cerevisiae. Microbiology 164: 464-474. doi:10.1099/mic.0 .000635

Bolukbasi MF, Gupta A, Oikemus S, Derr AG, Garber M, Brodsky MH, Zhu LJ, Wolfe SA. 2015. DNA-binding-domain fusions enhance the targeting range and precision of Cas9. Nat Methods 12: 1150-1156. doi:10.1038/nmeth.3624

Bondy-Denomy J. 2018. Protein inhibitors of CRISPR-Cas9. ACS Chem Biol 13: 417-423. doi:10.1021/acschembio.7b00831

Bondy-Denomy J, Pawluk A, Maxwell KL, Davidson AR. 2013. Bacteriophage genes that inactivate the CRISPR/Cas bacterial immune system. Nature 493: 429-432. doi:10.1038/nature11723

Bubeck F, Hoffmann MD, Harteveld Z, Aschenbrenner S, Bietz A, Waldhauer MC, Börner K, Fakhiri J, Schmelas C, Dietz L, et al. 2018. Engineered anti-CRISPR proteins for optogenetic control of CRISPR-Cas9. Nat Methods 15: 924-927. doi:10.1038/ s41592-018-0178-9

Cho SW, Kim S, Kim JM, Kim J-S. 2013. Targeted genome engineering in human cells with the Cas9 RNA-guided endonuclease. Nat Biotechnol 31: 230-232. doi:10.1038/nbt.2507

Cong L, Ran FA, Cox D, Lin S, Barretto R, Habib N, Hsu PD, Wu X, Jiang W, Marraffini LA, et al. 2013. Multiplex genome engineering using CRISPR/Cas systems. Science 339: 819-823. doi:10.1126/ science. 1231143

Deltcheva E, Chylinski K, Sharma CM, Gonzales K, Chao Y, Pirzada ZA, Eckert MR, Vogel J, Charpentier E. 2011. CRISPR RNA maturation by trans-encoded small RNA and host factor RNase III. Nature 471: 602. doi:10.1038/nature09886

Dong D, Guo M, Wang S, Zhu Y, Wang S, Xiong Z, Yang J, Xu Z, Huang Z. 2017. Structural basis of CRISPR-SpyCas9 inhibition by an anti-CRISPR protein. Nature 546: 436-439. doi:10.1038/ nature22377

Edraki A, Mir A, Ibraheim R, Gainetdinov I, Yoon Y, Song C-Q, Cao Y, Gallant J, Xue W, Rivera-Pérez JA, et al. 2018. A compact, highaccuracy Cas9 with a dinucleotide PAM for in vivo genome editing. Mol Cell 73: 714-726.e4. doi:10.1016/j.molcel.2018.12.003

Fukuhara T, Kambara H, Shiokawa M, Ono C, Katoh H, Morita E, Okuzaki D, Maehara Y, Koike K, Matsuura Y. 2012. Expression of microRNA miR-122 facilitates an efficient replication in nonhepatic cells upon infection with hepatitis C virus. J Virol 86: 7918-7933. doi:10.1128/JVI.00567-12

Gao G, Vandenberghe LH, Alvira MR, Lu Y, Calcedo R, Zhou X, Wilson JM. 2004. Clades of adeno-associated viruses are widely disseminated in human tissues. J Virol 78: 6381-6388. doi:10 .1128/JVI.78.12.6381-6388.2004

Garneau JE, Dupuis M-Ė, Villion M, Romero DA, Barrangou R, Boyaval $P$, Fremaux $C$, Horvath $P$, Magadán AH, Moineau S. 2010. The CRISPR/Cas bacterial immune system cleaves bacteriophage and plasmid DNA. Nature 468: 67-71. doi:10.1038/ nature09523

Harrington LB, Doxzen KW, Ma E, Liu J-J, Knott GJ, Edraki A, Garcia B, Amrani N, Chen JS, Cofsky JC, et al. 2017. A broad-spectrum inhibitor of CRISPR-Cas9. Cell 170: 1224-1233.e15. doi:10.1016/j .cell.2017.07.037

Hinderer C, Katz N, Buza EL, Dyer C, Goode T, Bell P, Richman LK, Wilson JM. 2018. Severe toxicity in nonhuman primates and piglets following high-dose intravenous administration of an adenoassociated virus vector expressing human SMN. Hum Gene Ther 29: 285-298. doi:10.1089/hum.2018.015

Hirosawa M, Fujita Y, Parr CJC, Hayashi K, Kashida S, Hotta A, Woltjen K, Saito H. 2017. Cell-type-specific genome editing with a microRNA-responsive CRISPR-Cas9 switch. Nucleic Acids Res 45: e118. doi:10.1093/nar/gkx309

Hirosawa M, Fujita Y, Saito H. 2019. Cell-type-specific CRISPR activation with microRNA-responsive AcrllA4 switch. ACS Synth Biol 8: 1575-1582. doi:10.1021/acssynbio.9b00073

Hoffmann MD, Aschenbrenner S, Grosse S, Rapti K, Domenger C, Fakhiri J, Mastel M, Börner K, Eils R, Grimm D, et al. 2019. Cell-specific CRISPR-Cas9 activation by microRNA-dependent expression of anti-CRISPR proteins. Nucleic Acids Res 47: e75. doi:10.1093/ nar/gkz271

Horak M, Novak J, Bienertova-Vasku J. 2016. Muscle-specific microRNAs in skeletal muscle development. Dev Biol 410: 1-13. doi:10.1016/j.ydbio.2015.12.013

Hwang WY, Fu Y, Reyon D, Maeder ML, Tsai SQ, Sander JD, Peterson RT, Yeh J-RJ, Joung JK. 2013. Efficient genome editing in zebrafish using a CRISPR-Cas system. Nat Biotechnol 31: 227229. doi:10.1038/nbt.2501

Hynes AP, Rousseau GM, Lemay M-L, Horvath P, Romero DA, Fremaux C, Moineau S. 2017. An anti-CRISPR from a virulent streptococcal phage inhibits Streptococcus pyogenes Cas9. Nat Microbiol 2: 1374-1380. doi:10.1038/s41564-017-0004-7

Hynes AP, Rousseau GM, Agudelo D, Goulet A, Amigues B, Loehr J, Romero DA, Fremaux C, Horvath P, Doyon Y, et al. 2018. Widespread anti-CRISPR proteins in virulent bacteriophages inhibit a range of Cas9 proteins. Nat Commun 9: 2919. doi:10 .1038/s41467-018-05092-w 
Ibraheim R, Song C-Q, Mir A, Amrani N, Xue W, Sontheimer EJ. 2018. All-in-one adeno-associated virus delivery and genome editing by Neisseria meningitidis Cas9 in vivo. Genome Biol 19: 137. doi:10 .1186/s13059-018-1515-0

Ivics Z, Hackett PB, Plasterk RH, Izsvák Z. 1997. Molecular reconstruction of Sleeping Beauty, a Tc1-like transposon from fish, and its transposition in human cells. Cell 91: 501-510. doi:10.1016/ S0092-8674(00)80436-5

Jiang J, Zhang L, Zhou X, Chen X, Huang G, Li F, Wang R, Wu N, Yan Y, Tong C, et al. 2016. Induction of site-specific chromosomal translocations in embryonic stem cells by CRISPR/Cas9. Sci Rep 6: 21918. doi:10.1038/srep21918

Jinek M, Chylinski K, Fonfara I, Hauer M, Doudna JA, Charpentier E. 2012. A programmable dual-RNA-guided DNA endonuclease in adaptive bacterial immunity. Science 337: 816-821. doi:10 $.1126 /$ science. 1225829

Jinek M, East A, Cheng A, Lin S, Ma E, Doudna J. 2013. RNAprogrammed genome editing in human cells. eLife 2: e00471. doi:10.7554/eLife.00471

Jonas S, Izaurralde E. 2015. Towards a molecular understanding of microRNA-mediated gene silencing. Nat Rev Genet 16: $421-$ 433. doi:10.1038/nrg3965

Komor AC, Badran AH, Liu DR. 2017. CRISPR-based technologies for the manipulation of eukaryotic genomes. Cell 169: 559. doi:10 .1016/j.cell.2017.04.005

Kosicki M, Tomberg K, Bradley A. 2018. Repair of double-strand breaks induced by CRISPR-Cas9 leads to large deletions and complex rearrangements. Nat Biotechnol 36: 765-771. doi:10.1038/ nbt.4192

Lagos-Quintana M, RauhutR, Yalcin A, Meyer J, Lendeckel W, Tuschl T. 2002. Identification of tissue-specific microRNAs from mouse. Curr Biol 12: 735-739. doi:10.1016/S0960-9822(02)00809-6

Lee J, Mir A, Edraki A, Garcia B, Amrani N, Lou HE, Gainetdinov I, Pawluk A, Ibraheim R, Gao XD, et al. 2018. Potent Cas9 inhibition in bacterial and human cells by AcrllC4 and AcrllC5 anti-CRISPR proteins. mBio 9. doi:10.1128/mBio.02321-18

Liu XS, Wu H, Krzisch M, Wu X, Graef J, Muffat J, Hnisz D, Li CH, Yuan B, Xu C, et al. 2018. Rescue of fragile $X$ syndrome neurons by DNA methylation editing of the FMR1 gene. Cell 172: 979992.e6. doi:10.1016/j.cell.2018.01.012

Maddalo D, Manchado E, Concepcion CP, Bonetti C, Vidigal JA, Han Y-C, Ogrodowski P, Crippa A, Rekhtman N, de Stanchina E, et al. 2014. In vivo engineering of oncogenic chromosomal rearrangements with the CRISPR/Cas9 system. Nature 516: 423427. doi:10.1038/nature13902

Makarova KS, Wolf YI, Koonin EV. 2018. Classification and nomenclature of CRISPR-Cas systems: where from here? CRISPR J 1: 325336. doi:10.1089/crispr.2018.0033

Mali P, Yang L, Esvelt KM, Aach J, Guell M, DiCarlo JE, Norville JE, Church GM. 2013. RNA-guided human genome engineering via Cas9. Science 339: 823-826. doi:10.1126/science.1232033

Marino ND, Zhang JY, Borges AL, Sousa AA, Leon LM, Rauch BJ, Walton RT, Berry JD, Joung JK, Kleinstiver BP, et al. 2018. Discovery of widespread type I and type V CRISPR-Cas inhibitors. Science 362: 240-242. doi:10.1126/science.aau5174

Marshall R, Maxwell CS, Collins SP, Jacobsen T, Luo ML, Begemann MB, Gray BN, January E, Singer A, He Y, et al. 2018. Rapid and scalable characterization of CRISPR technologies using an E. coli cell-free transcription-translation system. Mol Cell 69: 146-157.e3. doi:10.1016/j.molcel.2017.12.007

Nakai H, Fuess S, Storm TA, Muramatsu S-I, Nara Y, Kay MA. 2005. Unrestricted hepatocyte transduction with adeno-associated virus serotype 8 vectors in mice. J Virol 79: 214-224. doi:10.1128/JVI $.79 .1 .214-224.2005$
Nakamura M, Srinivasan $P$, Chavez M, Carter MA, Dominguez AA, La Russa M, Lau MB, Abbott TR, Xu X, Zhao D, et al. 2019. AntiCRISPR-mediated control of gene editing and synthetic circuits in eukaryotic cells. Nat Commun 10: 194. doi:10.1038/s41467018-08158-x

Pawluk A, Amrani N, Zhang Y, Garcia B, Hidalgo-Reyes Y, Lee J, Edraki A, Shah M, Sontheimer EJ, Maxwell KL, et al. 2016. Naturally occurring off-switches for CRISPR-Cas9. Cell 167: 1829-1838.e9. doi:10.1016/j.cell.2016.11.017

Rauch BJ, Silvis MR, Hultquist JF, Waters CS, McGregor MJ, Krogan NJ, Bondy-Denomy J. 2017. Inhibition of CRISPR-Cas9 with bacteriophage proteins. Cell 168: 150-158.e10. doi:10 .1016/j.cell.2016.12.009

Senís E, Fatouros C, Große S, Wiedtke E, Niopek D, Mueller A-K, Börner K, Grimm D. 2014. CRISPR/Cas9-mediated genome engineering: an adeno-associated viral (AAV) vector toolbox. Biotechnol J 9: 1402-1412. doi:10.1002/biot.201400046

Shin J, Jiang F, Liu J-J, Bray NL, Rauch BJ, Baik SH, Nogales E, BondyDenomy J, Corn JE, Doudna JA. 2017. Disabling Cas9 by an antiCRISPR DNA mimic. Sci Adv 3: e1701620. doi:10.1126/sciadv .1701620

Uribe RV, van der Helm E, Misiakou M-A, Lee S-W, Kol S, Sommer MOA. 2019. Discovery and characterization of Cas9 inhibitors disseminated across seven bacterial phyla. Cell Host Microbe 25: 233-241.e5. doi:10.1016/j.chom.2019.01.003

van Gent M, Gack MU. 2018. Viral anti-CRISPR tactics: no success without sacrifice. Immunity 49: 391-393. doi:10.1016/j.immuni .2018 .08 .023

Walther W, Stein U. 1996. Cell type specific and inducible promoters for vectors in gene therapy as an approach for cell targeting. J Mol Med 74: 379-392. doi:10.1007/BF00210632

Wang X-W, Hu L-F, Hao J, Liao L-Q, Chiu Y-T, Shi M, Wang Y. 2019. A microRNA-inducible CRISPR-Cas9 platform serves as a microRNA sensor and cell-type-specific genome regulation tool. Nat Cell Biol 21: 522-530. doi:10.1038/s41556-019-0292-7.

Watters KE, Fellmann C, Bai HB, Ren SM, Doudna JA. 2018. Systematic discovery of natural CRISPR-Cas12a inhibitors. Science 362: 236-239. doi:10.1126/science.aau5138

Xie J, Xie Q, Zhang H, Ameres SL, Hung J-H, Su Q, He R, Mu X, Seher Ahmed S, Park S, et al. 2011. MicroRNA-regulated, systemically delivered rAAV9: a step closer to CNS-restricted transgene expression. Mol Ther 19: 526-535. doi:10.1038/mt.2010.279

Xue W, Meylan E, Oliver TG, Feldser DM, Winslow MM, Bronson R, Jacks T. 2011. Response and resistance to NF- $\mathrm{KB}$ inhibitors in mouse models of lung adenocarcinoma. Cancer Discov 1: 236247. doi:10.1158/2159-8290.CD-11-0073

Xue W, Chen S, Yin H, Tammela T, Papagiannakopoulos T, Joshi NS, Cai W, Yang G, Bronson R, Crowley DG, et al. 2014. CRISPR-mediated direct mutation of cancer genes in the mouse liver. Nature 514: 380-384. doi:10.1038/nature13589

Yang H, Patel DJ. 2017. Inhibition mechanism of an anti-CRISPR suppressor AcrllA4 targeting SpyCas9. Mol Cell 67: 117-127.e5. doi:10.1016/j.molcel.2017.05.024

Zhang G, Budker V, Wolff JA. 1999. High levels of foreign gene expression in hepatocytes after tail vein injections of naked plasmid DNA. Hum Gene Ther 10: 1735-1737. doi:10.1089/ 10430349950017734

Zhu Y, Gao A, Zhan Q, Wang Y, Feng H, Liu S, Gao G, Serganov A, Gao P. 2019. Diverse mechanisms of CRISPR-Cas9 inhibition by type II-C anti-CRISPR proteins. Mol Cell 74: 296-309. doi:10 .1016/j.molcel.2019.01.038

Zincarelli C, Soltys S, Rengo G, Rabinowitz JE. 2008. Analysis of AAV serotypes 1-9 mediated gene expression and tropism in mice after systemic injection. Mol Ther 16: 1073-1080. doi:10.1038/mt.2008 .76 

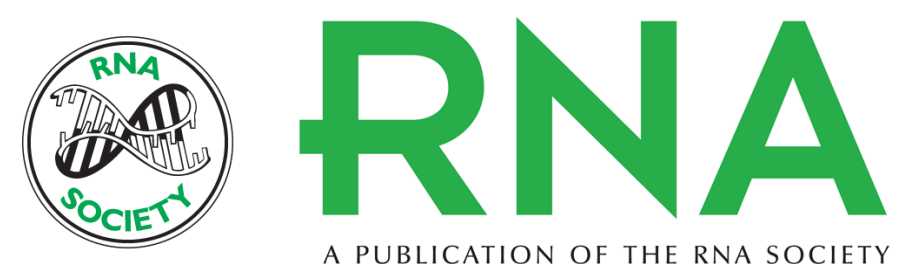

A PUBLICATION OF THE RNA SOCIETY

\section{Tissue-restricted genome editing in vivo specified by microRNA-repressible anti-CRISPR proteins}

Jooyoung Lee, Haiwei Mou, Raed Ibraheim, et al.

RNA 2019 25: 1421-1431 originally published online August 22, 2019

Access the most recent version at doi:10.1261/rna.071704.119

\section{Supplemental http://rnajournal.cshlp.org/content/suppl/2019/08/22/rna.071704.119.DC1 \\ Material}

References This article cites 59 articles, 12 of which can be accessed free at: http://rnajournal.cshlp.org/content/25/11/1421.full.html\#ref-list-1

Creative This article is distributed exclusively by the RNA Society for the first 12 months after the Commons License full-issue publication date (see http://rnajournal.cshlp.org/site/misc/terms.xhtml). After 12 months, it is available under a Creative Commons License (Attribution-NonCommercial 4.0 International), as described at http://creativecommons.org/licenses/by-nc/4.0/.

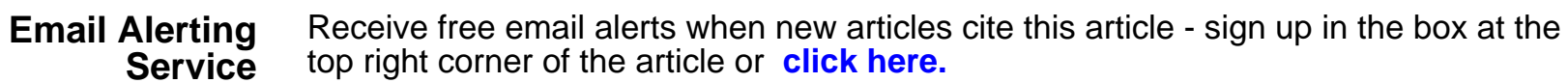

To subscribe to RNA go to:

http://rnajournal.cshlp.org/subscriptions 